\title{
Differences in Electrophysiologic Values between Preoperative and Intraoperative Neurophysiologic Monitoring
}

\author{
Da-sol KIM${ }^{1}$, Ha-Young $\mathrm{CHOI}^{2}$, Gi-Wook KIM${ }^{1}$, Jeong-Hwan SEO ${ }^{1}$, Yu Hui WON ${ }^{1,3}$ \\ ${ }^{1}$ Jeonbuk National University Medical School and Research Institute of Clinical Medicine of Jeonbuk National University - Biomedical Research \\ Institute of Jeonbuk National University Hospital, Department of Physical Medicine and Rehabilitation, Jeonju, South Korea \\ ${ }^{2}$ Jeonbuk National University Medical School, Department of Neurosurgery, Jeonju, South Korea \\ ${ }^{3}$ Rehabailitation Institute of Neuromuscular Disease, Yonsei University College of Medicine, Seoul, Korea
}

Corresponding author: Yu Hui WON wonyh@jbnu.ac.kr

\section{ABSTRACT}

AIM: To compare preoperative and intraoperative electrophysiologic values.

MATERIAL and METHODS: A retrospective medical record review was conducted on patients who underwent brain and spine surgery between January 2014 and July 2018. Patients underwent preoperative electrophysiologic monitoring within a week before the operation and intraoperative monitoring during the surgery. Monitoring parameters included the onset latency (msec) and amplitude $(\mathrm{mV})$ of motor evoked potential (MEP), somatosensory evoked potential (SEP), electroneuronography, and brainstem auditory evoked potential (BAEP).

RESULTS: The latency of the MEP and SEP were significantly prolonged while the amplitude was decreased during operation. Specifically, patients with abnormal findings in their preoperative assessments showed more prominent differences compared to those with normal findings. However, there was no significant difference between preoperative and intraoperative results based on which side of the hemisphere was affected or unaffected. Unlike the MEP and SEP parameters, there were no significant changes in the facial electroneuronography and BAEP parameters.

CONCLUSION: There were differences in the preoperative and intraoperative monitoring parameters. Further studies are necessary to understand the underlying mechanisms behind these changes during surgery.

KEYWORDS: Intraoperative neurophysiologic monitoring, Motor evoked potential, Somatosensory evoked potential, Brain stem auditory evoked potential, Electroneuronography

ABBREVIATIONS: MEP: Motor evoked potential, SEP: Somatosensory evoked potential, BAEP: Brainstem auditory evoked potential, IONM: Intraoperative neurophysiologic monitoring, PONM: Preoperative neurophysiologic monitoring, TIVA: Total intravenous anesthesia, TOF: Train-of-four, FDI: First dorsal interossei, APB: Abductor pollicis brevis, BB: Biceps brachii, DEL: Deltoid, TA: Tibialis anterior, AH: Abductor hallucis, LSR: Lateral speed response, EMG: Electromyographic, TIVA: Total intravenous anesthesia, IA: Inhalation anesthesia

Da-sol KIM (10): 0000-0002-7745-978X

Ha-Young CHOI (D) : 0000-0002-6518-247X

Gi-Wook KIM (1) : 0000-0002-1628-8382
Jeong-Hwan SEO (1) : 0000-0002-6915-6674

Yu Hui WON (1) : 0000-0003-2007-9652 


\section{- INTRODUCTION}

$\mathrm{I}$ ntraoperative neurophysiologic monitoring (IONM) is an efficient method of providing anatomical and functional information on adjacent nervous systems to surgeons during operations, thereby helping to prevent neurological complications $(7,13)$. Nevertheless, the low success rate of motor evoked potentials in patients with neurologic deficits and the false-positive and negative results have made IONM difficult to precisely understand for neurophysiologists and neurosurgeons $(3,4,6)$. However, preoperative neurophysiologic monitoring (PONM) can solve these problems and improve accuracy $(2,10,11)$. For example, we can detect the optimal stimulating location before the operation, which might increase the success rate and improve the reliability of the IONM data. Moreover, PONM gives individualized reference data that might be useful to identify false-positive and negative results.

However, PONM has several limitations. The use of a neuromuscular blocker and inhalation anesthesia during an operation can result in a larger difference between IONM and PONM values. Moreover, conducting IONM and PONM using different machines can result in a difference in the recorded values $(10,11)$. Additionally, although individualized reference data with predictive ability can be obtained from PONM, we still do not fully understand whether the differences between PONM and IONM values are normal. Therefore, further studies are required on PONM to investigate these differences and determine reference values.

In this study, PONM and IONM values were examined in all patients who underwent neurosurgical treatment, including aneurysmal neck clipping, brain and spine tumor mass removal, carotid endarterectomy, bypass surgery, and microvascular decompression. The aims of this study were to determine the pattern of change of the electrophysiologic data before and during the operations, determine the normative differences, and ultimately to understand how to efficiently use PONM to improve the accuracy of IONM.

\section{- MATERIAL and METHODS}

\section{Study Design}

Demographic data and electrophysiologic values were retrospectively collected from patients who underwent brain and neurovascular surgery between January 2014 and July 2018, such as aneurysmal neck clipping, tumor mass removal, carotid endarterectomy, bypass surgery, cerebral artery ligation, and spine surgery. All patients were subjected to preoperative monitoring within a week before the operation as well as intraoperative monitoring, in which the baseline data is usually measured prior to dura opening during the operation. Data on these monitoring processes were collected from medical records for data analysis. This study analyzed the differences in the electrophysiologic parameters obtained from PONM and IONM, including the latency and amplitude. The study was approved by the local institutional review board, and informed consent was waived due to its retrospective study design.

\section{Anesthesia Protocol}

According to standard protocol, total intravenous anesthesia (TIVA) using propofol and opiate (remifentanil) infusions, with or without dexmedetomidine infusion, was recommended as the optimum regimen. Inhalational anesthesia (IA), including sevoflurane or desflurane, was administered either in combination with TIVA or exclusively at the anesthesiologist's discretion. Short-acting muscle relaxant drugs, such as rocuronium, were infused only during induction. Each patient's blood pressure and body temperature was maintained above $60 \mathrm{mmHg}$ and between 34.0 and $37.0^{\circ} \mathrm{C}$, respectively. Furthermore, the train-of-four (TOF) test, which measures electromyographic activity elicited by ulnar nerve stimulation to predict the effect of the neuromuscular blockade, was performed. The number of twitches observed was calculated as a percentage of blocked receptors, and the anesthesiologists tried to maintain the number of twitches to more than 2 or 3 twitches, which is equivalent to a receptor blockade percentage under $75 \sim 80 \%$ (5).

\section{Preoperative Neurophysiologic Monitoring}

The preoperative somatosensory evoked potential (SEP) was assessed using Medelec Synergy (Cardinal Health Inc., USA). Surface electrodes for stimulation were bilaterally localized between the tendons of the palmaris longus and the flexor carpi radialis for the median nerve SEP, between the medial malleolus and the Achilles tendon for the tibial nerve SEP, and at the popliteal fossa for the peroneal nerve SEP (8). The stimulation intensity was adjusted to be just enough to produce a visible twitch, and the stimulating frequency was set to a range between 4 and $7 \mathrm{~Hz}(8)$. The recording electrodes were placed on the C3' or C4' scalp region for the median nerve and the Cz' scalp area for the tibial and peroneal nerve. The ground electrodes were placed between the stimulating and recording electrodes for the median nerve, around the calf for the tibial nerve, and on the posterior mid-thigh for the peroneal nerve. For the motor evoked potential (MEP), the Medtronic Keypoint ${ }^{\circledR}$ was used (Medtronic Inc., Skovlunde, Denmark) with 70-mm, figure 8-shaped coils, which marked the optimal stimulus point, also known as "the hot spot", of the motor cortex on the right and left. The stimulation intensity was adjusted at $110 \%$ to the lowest resting motor threshold, and an $\mathrm{x}$-line was drawn to indicate the hot spot until the operation. The surface recording electrodes were localized at the bilateral first dorsal interossei (FDI), abductor pollicis brevis (APB), biceps brachii (BB), deltoid (DEL), tibialis anterior (TA), and abductor hallucis $(\mathrm{AH})$ muscles.

Facial electroneuronography and brain stem auditory evoked potentials (BAEP) were assessed using Medelec Synergy (Cardinal Health Inc., USA). For facial electroneuronography, surface stimulation was performed below the ear lobe, using a 10 to 30 msec pulse wave of approximately $90-100 \mathrm{mV}$ intensity, and the values were obtained from the frontalis and orbicularis oculi muscles using surface electrodes. To obtain the lateral spread response (LSR), the zygomatic branch of the facial nerve was stimulated with a 10 to $30 \mathrm{msec}$ pulse wave of $10 \sim 17 \mathrm{mV}$ intensity, which marked the optimal site to maximize the amplitude with the minimum stimulating intensity. 
Kim D. et al: Preoperative and Intraoperative Neurophysiologic Monitoring

In these cases, the recording electrodes were placed in the orbicularis oris and mentalis muscles. In BAEP, the stimulation was started at $75 \mathrm{~dB}$ and was gradually increased depending on the patients' response (8 to $10 \mathrm{~Hz}$, averaging 400 times), and the recording electrodes were bilaterally placed over the earlobes (A1 and A2).

\section{Intraoperative Neurophysiologic Monitoring (IONM)}

All patients who underwent IONM underwent SEP, MEP, BAEP, and facial LSR assessments even in cases in which PONM showed abnormal findings or even an absence of a response. We continuously observed the real-time graph to capture the best baseline. At the same time, the results of the TOF tests were checked to minimize the negative effects of neuromuscular blockades. All instances of IONM were performed using the ISIS IOM System (Inomed Inc., Germany). In IONM, the SEP was obtained through an almost identical setting to that of the PONM. In MEP, the pre-indicated motor hot spot was stimulated with a $0.1-\mathrm{msec}$ pulse wave of a $40 \sim 220 \mathrm{~mA}$ intensity and a $0.5 \sim 2-\mathrm{Hz}$ frequency and the results were recorded for the FDI, APB, BB, DEL, TA or AH muscles, depending on the type and level of operation. Facial electroneuronography was also assessed by stimulating the previously marked optimal site with a $0.2 \mathrm{msec}$ pulse wave of a $3 \sim 30 \mathrm{~Hz}$ frequency and a $10 \sim 45 \mathrm{~mA}$ intensity, while the BAEP was assessed via a stimulation of $90 \sim 100 \mathrm{~dB}$ and 14.3 $\sim 18.2 \mathrm{~Hz}$, and by averaging 400 times.

\section{Statistical Analysis}

For statistical analysis, SPSS software (version 18.0 for Windows; SPSS Inc., Chicago, USA) was used. The data were analyzed using the normality test, and for comparison of the neurophysiologic parameters between PONM and IONM, we used the paired t-test in the normality groups and Wilcoxon signed rank test in the non-normality groups. An independent $t$-test and Mann-Whitney $U$ test were used to identify whether there were any differences between the normal and abnormal group in obtaining PONM, and whether the unaffected and affected hemispheres in the patients caused statistically significant differences. A P-value of less than 0.05 was considered statistically significant.

\section{RESULTS}

\section{Demographic Features}

In this study, a total of 123 patients with a mean age of 57-years-old (ranging from approximately 7 to 77 -years-old) were subjected to IONM at our Department of Neurosurgery. The PONM and IONM were performed in patients undergoing brain or spine operations, using MEP/SEP or facial electroneuronography/BAEP (Table I). In the brain operations, including the first and third group, the right hemisphere was more affected than the left hemisphere in $52(58.4 \%)$ patients. The patients with lumbar lesions in the spine operation group were more than those with cervical and thoracic lesions (cervical: thoracic: lumbar ratio $=14.7 \%: 41.7 \%: 44.1 \%$ ). The uses of IA were higher in the spine operation (23.5\%) than in the brain operation groups. The TOF (\%) was above $75 \%$ in the third group but was $63.54 \%$ in the other groups.

\section{Comparison of Neurophysiologic Parameters in MEP and SEP Between PONM and IONM}

For the MEP and SEP, the mean values of the latency and amplitude of muscles in the upper and lower extremities in PONM and IONM for both brain and spine operations are shown in Table II. The latency of these muscles in IONM was mostly delayed in both MEP and SEPs, ranging from an average of 1.86 to $4.41 \mathrm{msec}$, while the MEP amplitudes (of mean 36 to $38 \%$ ) in IONM decreased compared to PONM. The differences between IONM and PONM $(\Delta)$ were significant in terms of the latency and amplitude of MEP/SEP $(p<0.001)$. In the MEP in IONM, the onset latency was more prolonged in muscles in the lower extremities than those in the upper extremities, while the amplitude was more decreased in muscles in the upper extremities. Unlike the MEP, the latency of the SEP during the operation was more increased at the upper extremities.

Comparison of the Differences in MEP and SEP Between the Normal and Abnormal Group in PONM

The differences in values of the latency and amplitude between PONM and IONM were compared between the normal and abnormal group, which were determined based on the PONM measurements (Table III). Except for the tibial nerve SEP measurements, the differences in the latency and amplitude in the abnormal group were greater than in the normal group. For the MEP, the latency in both the normal and abnormal group was higher in the lower extremities than in the upper extremities, which was similarly observed in the SEPS of the normal group. However, in the SEPs of the abnormal group, the differences in the latency at the upper extremities were greater than at the lower extremities. The differences in MEP latency and amplitude were not significantly different between the normal and abnormal group; however, there was a significant difference in the SEP latency between the normal and abnormal group. The latency differences in the tibial SEP in the abnormal group did not show any increasing or decreasing pattern or trend, unlike latency differences in tibial MEP and median SEP.

\section{Comparison of the Differences in MEP and SEP Between the Unaffected and Affected Group}

The neurophysiologic parameters obtained during brain operations were separated according to the unaffected and affected hemisphere (Table IV). Fifty-two patients were more affected in the right than the left hemisphere (Right: Left hemisphere $=52: 37$ ) (Table I). The differences in the latency and amplitude of the MEPs and SEPs between PONM and IONM showed no significant difference between the unaffected and affected hemispheres (Table IV), and the results showed that both the MEP and SEP latency in IONM were delayed while the MEP amplitude in IONM was decreased.

\section{Comparison of Electroneuronography and BAEP Between PONM and IONM}

In electroneuronography, the latency and amplitude of the facial muscles and the latency of the BAEP were measured, as shown in Table V. A total of 38 patients were subjected 
Kim D. et al: Preoperative and Intraoperative Neurophysiologic Monitoring

Table I: Demographic Characteristics of Patient Population

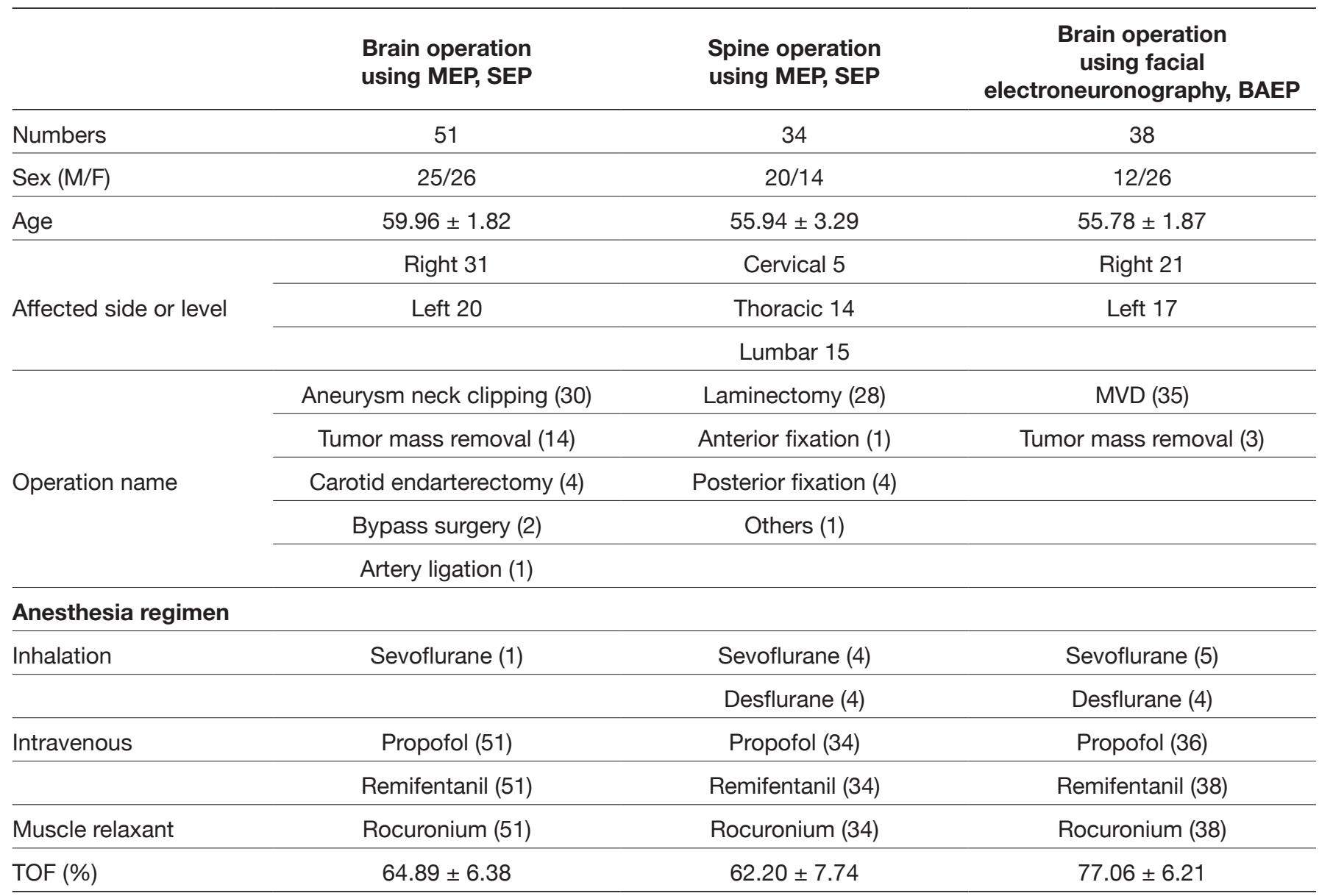

MEP: Motor evoked potential, SEP: Somatosensory evoked potential, AEP: Auditory evoked potential, M: Male, F: Female, MVD: Microvascular decompression, TOF: Train of four. Age and TOF were expressed as mean \pm standard deviation.

Table II: Comparison of Motor Evoked Potential and Somatosensory Evoked Potentials in Preoperative and Intraoperative Neurophysiologic Monitoring

\begin{tabular}{|c|c|c|c|c|c|}
\hline & $\mathbf{N}(\mathbf{n})$ & PONM & IONM & $\Delta$ & $\mathbf{p}$ \\
\hline \multicolumn{6}{|l|}{ MEP } \\
\hline FDI/APB latency (msec) & \multirow{2}{*}{$45(90)$} & $22.00 \pm 1.26$ & $25.13 \pm 3.59$ & $3.24 \pm 3.40$ & $<0.001^{2)}$ \\
\hline FDI/APB amplitude (mV) & & $2.90 \pm 2.43$ & $1.91 \pm 1.57$ & $-1.03 \pm 2.38$ & $<0.001^{2)}$ \\
\hline TA latency (msec) & \multirow{2}{*}{$31(62)$} & $30.55 \pm 2.44$ & $34.97 \pm 4.79$ & $4.41 \pm 3.70$ & $<0.001^{1)}$ \\
\hline TA amplitude (mV) & & $0.95 \pm 0.65$ & $0.58 \pm 0.76$ & $-0.36 \pm 0.99$ & $<0.001^{2)}$ \\
\hline \multicolumn{6}{|l|}{ SEP } \\
\hline Median N19 (msec) & \multirow{2}{*}{$57(114)$} & $19.25 \pm 1.60$ & $21.61 \pm 4.78$ & $2.36 \pm 4.34$ & $<0.001^{2)}$ \\
\hline Median P23 (msec) & & $24.97 \pm 2.10$ & $29.14 \pm 6.16$ & $4.16 \pm 5.89$ & $<0.001^{2)}$ \\
\hline Tibial P1 (msec) & \multirow{2}{*}{$61(122)$} & $41.17 \pm 4.52$ & $43.04 \pm 6.02$ & $1.86 \pm 5.87$ & $<0.001^{2)}$ \\
\hline Tibial N1 (msec) & & $49.24 \pm 0.49$ & $53.21 \pm 0.61$ & $3.86 \pm 7.71$ & $<0.001^{2)}$ \\
\hline
\end{tabular}

MEP: Motor evoked potential, SEP: Somatosensory evoked potential, FDI: 1st digit interosseous, APB: abductor pollicis brevis, TA: tibialis anterior, $\Delta$ : intraoperative value - preoperative value, $\mathbf{N}$ : number of examined patients, $\boldsymbol{n}$ : number of collected data, PONM: Preoperative neurophysiologic monitoring, IONM: Intraoperative neurophysiologic monitoring. 1) were analyzed by paired t-test and 2) were by Wilcoxon signed rank test. Latency and amplitude were expressed as mean \pm standard deviation. 
to electrophysiologic evaluations during PONM; however, during IONM, there was no response from 1 patient for BAEP, nor from 4 for frontalis, 9 for oculi, 24 for oris, and 16 for mentalis electroneuronography. The amplitudes of the oris and mentalis muscles during the evaluation of LSR were very low even during PONM and a lot of data were lost during IONM. Although the sample size was small, the latency of the orbicularis oris significantly decreased to about 2.07 msec during operation $(p=0.008)$, while the amplitude of the mentalis significantly decreased by $28 \%(p=0.011)$. However, there was no significant difference in the other parameters obtained during PONM and IONM. There were statistical differences BAEP measurements of waves I and $\mathrm{V}$ between PONM and IONM; however, the differences of the latency in waves I, III, and V were all less than 1 msec (Table V).

Table III: Differences in Motor Evoked Potential and Somatosensory Evoked Potential Between the Normal and Abnormal Groups in PONM

\begin{tabular}{|c|c|c|c|c|}
\hline & $N(n)\left[{ }_{N} /{ }_{A B N}\right]$ & ${ }^{\Delta} \mathbf{N}$ & ${ }^{\triangle} \mathrm{ABN}$ & P-value \\
\hline \multicolumn{5}{|l|}{ MEP } \\
\hline FDI/APB latency (msec) & \multirow{2}{*}{$\begin{array}{l}45(90) \\
{[37 / 8]}\end{array}$} & $3.13 \pm 3.57$ & $3.76 \pm 2.44$ & $0.187^{2)}$ \\
\hline FDI/APB amplitude (mV) & & $-0.87 \pm 2.30$ & $-1.79 \pm 2.65$ & $0.254^{2)}$ \\
\hline TA latency (msec) & \multirow{2}{*}{$\begin{array}{c}31(62) \\
{[20 / 11]}\end{array}$} & $4.35 \pm 3.91$ & $4.56 \pm 3.24$ & $0.944^{2)}$ \\
\hline TA amplitude (mV) & & $-0.19 \pm 1.04$ & $-0.79 \pm 0.73$ & $0.045^{2)}$ \\
\hline \multicolumn{5}{|l|}{ SEP } \\
\hline Median N19 (msec) & \multirow{2}{*}{$\begin{array}{l}57(114) \\
{[40 / 17]}\end{array}$} & $1.74 \pm 2.24$ & $4.08 \pm 7.41$ & $<0.001^{2)}$ \\
\hline Median P23 (msec) & & $3.37 \pm 3.84$ & $6.40 \pm 9.27$ & $<0.001^{2)}$ \\
\hline Tibial P1 (msec) & \multirow{2}{*}{$\begin{array}{l}61(122) \\
{[39 / 22]}\end{array}$} & $2.32 \pm 4.40$ & $0.98 \pm 7.94$ & $0.609^{1)}$ \\
\hline Tibial N1 (msec) & & $4.63 \pm 5.66$ & $2.69 \pm 10.55$ & $0.001^{2)}$ \\
\hline
\end{tabular}

MEP: Motor evoked potential, SEP: Somatosensory evoked potential, FDI: 1st digit interosseous, APB: abductor pollicis brevis, TA: Tibialis anterior; ${ }^{\Delta} \mathbf{N}$ : Intraoperative value - preoperative value in normal group; ${ }^{\boldsymbol{A}} \boldsymbol{A B N}$ : intraoperative value - preoperative value in abnormal group, $\boldsymbol{N}$ : number of examined patients, $n$ : Number of collected data, N/ABN: Number of examined patients in normal group/abnormal group. 1) were analyzed by independent $t$-test and 2) were by Mann-Whitney $U$ test. Latency and amplitude were expressed as mean \pm standard deviation.

Table IV: Differences of Motor Evoked Potential and Somatosensory Evoked Potential Between the Unaffected and Affected Hemisphere in Patients with Brain Operation

\begin{tabular}{|c|c|c|c|c|}
\hline & $N(n)$ & $\triangle \mathbf{U A}$ & ${ }_{A}^{\Delta}$ & $\mathbf{p}$ \\
\hline \multicolumn{5}{|l|}{ MEP } \\
\hline FDI/APB latency(msec) & \multirow{2}{*}{$42(84)$} & $3.34 \pm 3.40$ & $3.30 \pm 3.51$ & $0.982^{2)}$ \\
\hline FDI/APB amplitude(mV) & & $-1.26 \pm 2.45$ & $-0.96 \pm 2.43$ & $0.932^{11}$ \\
\hline TA latency(msec) & \multirow{2}{*}{$20(40)$} & $4.64 \pm 3.28$ & $4.72 \pm 3.17$ & $0.693^{1)}$ \\
\hline TA amplitude(mV) & & $-0.42 \pm 0.69$ & $-0.17 \pm 1.28$ & $0.698^{2)}$ \\
\hline \multicolumn{5}{|l|}{ SEP } \\
\hline Median N19 (msec) & \multirow{2}{*}{$49(98)$} & $2.05 \pm 3.67$ & $1.98 \pm 4.08$ & $0.606^{2)}$ \\
\hline Median P23 (msec) & & $3.61 \pm 4.76$ & $3.81 \pm 5.84$ & $0.859^{2)}$ \\
\hline Tibial P1 (msec) & \multirow{2}{*}{$38(76)$} & $1.26 \pm 6.92$ & $1.75 \pm 6.59$ & $0.880^{2)}$ \\
\hline Tibial N1 (msec) & & $2.73 \pm 9.04$ & $3.54 \pm 7.88$ & $0.775^{2)}$ \\
\hline
\end{tabular}

MEP: Motor evoked potential, SEP: Somatosensory evoked potential, FDI: 1st digit interosseous, APB: abductor pollicis brevis, TA: Tibialis anterior, ${ }^{\Delta} \boldsymbol{U A}$ : Intraoperative value - preoperative value at the unaffected hemisphere, ${ }^{\boldsymbol{\Delta}} \boldsymbol{A}$ : Intraoperative value - preoperative value at the affected hemisphere; $\boldsymbol{N}$ : number of examined patients, $\boldsymbol{n}$ : Number of collected data. 1) were analyzed by independent $t$-test and 2) were by Mann-Whitney $U$ test. Latency and amplitude were expressed as mean \pm standard deviation. 
Kim D. et al: Preoperative and Intraoperative Neurophysiologic Monitoring

Table V: Comparison of Electroneuronography and Brain Stem Auditory Evoked Potential Studies in Preoperative and Intraoperative Neurophysiologic Monitoring

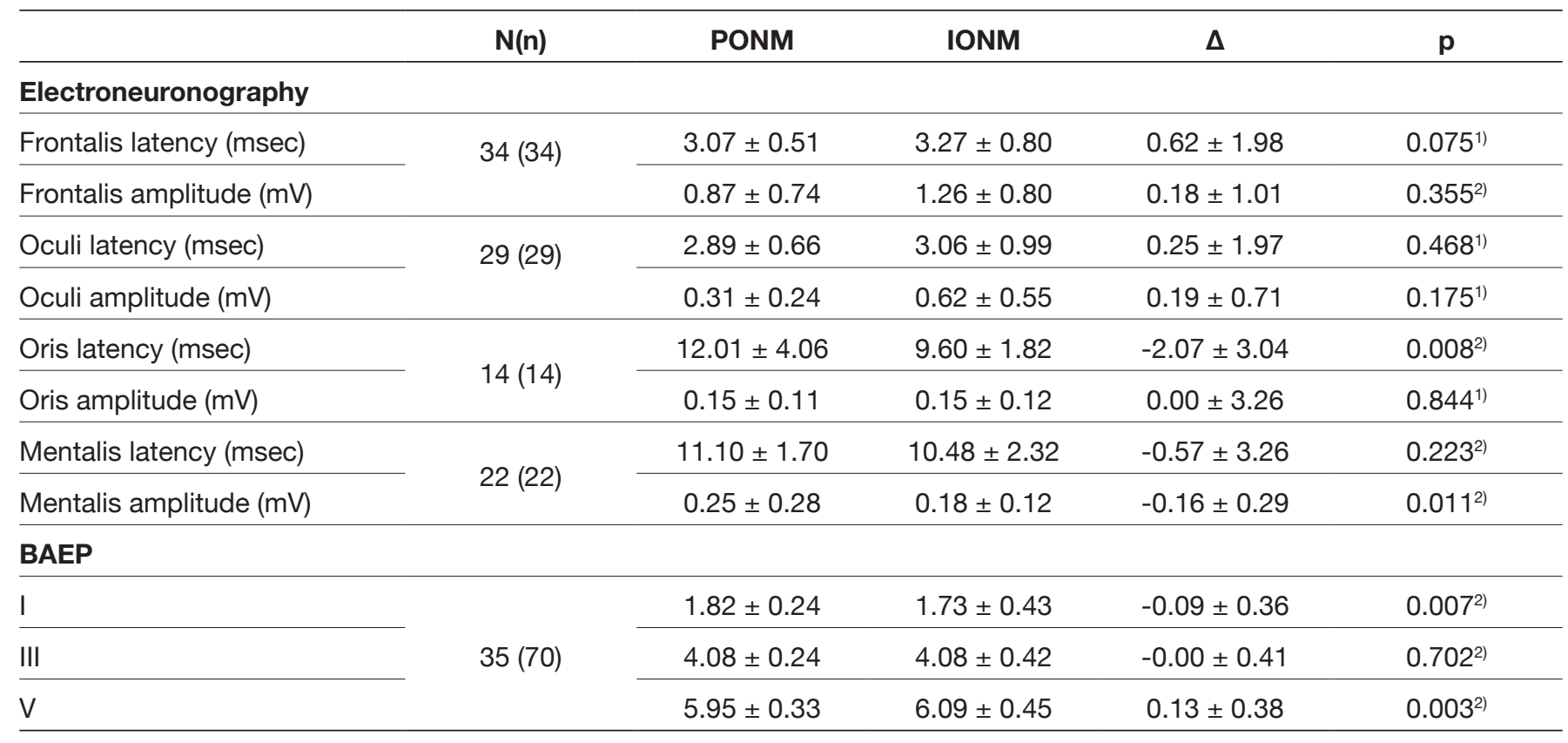

BAEP: Brain stem auditory evoked potential, Oculi: Orbicular oculi, Oris: Orbicular oris, $\Delta$ : Difference value between preoperation and during operation, $\mathbf{N}$ : number of examined patients, $\boldsymbol{n}$ : Number of collected data, PONM: Preoperative neurophysiologic monitoring, IONM: Intraoperative neurophysiologic monitoring. 1) were analyzed by paired t-test and 2) were by Wilcoxon signed rank test. Latency and amplitude were expressed as mean \pm standard deviation.

\section{DISCUSSION}

In this study, we compared and found significant differences between electrophysiologic parameters obtained during operations and those obtained during preoperative monitoring. Compared to the values obtained during PONM, the mean latency of the MEPs increased by more than $3 \mathrm{msec}$ while the mean amplitude decreased by approximately 36 to $38 \%$ during IONM. Moreover, there was a delayed latency in the SEPs of approximately $3 \mathrm{msec}$ in the median and tibial nerves. These changes were more significant in patients who showed abnormal findings during PONM, except for the SEPs in the tibial nerve. However, the side of the affected or unaffected hemisphere did not show any significant differences in the parameters obtained. Compared to the MEP and SEP, the difference in the electroneuronography and BAEP parameters between PONM and IONM was less significant.

Several factors have been shown to affect baseline values during intraoperative monitoring, including 1) anesthesia regimens, 2) surrounding environments, including body temperature, and blood pressure, and 3) individual factors, such as age, lesion location, and neurologic deficits (12). Specifically, the use of neuromuscular blockade, IA, and nitrous oxide has been shown to influence electrophysiologic monitoring. IA and nitrous oxide have been shown to have a dose-dependent effect on the mean amplitude values of transcranial electrical MEPs. These anesthetics have also been shown to increase the mean latency and decrease the amplitude values of SEPs and BAEPs, respectively. These effects are especially prominent in the SEPs for muscles in the lower extremities and in young children (12). Neuromuscular blockade can decrease the success rate of the responsiveness in MEPs and interfere with the monitoring of spontaneous electromyographic (EMG) activity and compound muscle action potentials. However, these agents can facilitate SEP monitoring by suppressing EMG artifacts (12). In light of this, anesthesiology guidelines have recommended that the TOF during IONM be maintained at more than 2 or 3 and $70 \%$ (12). Moreover, the use of propofol, which is one of the TIVAs, can cause a decrease in the MEP and SEP amplitudes; however, these influences are relatively smaller than those caused by IAs (12). Additionally, opioids can also be a proper anesthesia regimen alternative during intraoperative monitoring due to their minimal effect on electrophysiologic parameters (9, 12). A study reported that a one-degree decrease in body temperature caused a $3 \%$ increase in the latency and a $7 \%$ decline in the amplitude, while a decrease in mean blood pressure over $60 \mathrm{mmHg}$ caused a loss of SEP responsiveness. In addition to these environmental factors, demographic factors including age, lesion location, and neurologic deficits have also been shown to influence the success rate of MEPs. A previous study reported the success rates of MEPs as $55.0 \%$ in the under 7 years-old group and $70.2 \%$ in the over 7 years-old group, $51.0 \%$ for spine operations and $78.7 \%$ for brain operations, and $39.1 \%$ in patients with neurologic deficits and $78.8 \%$ in neurologically intact patients (1).

In this study, the latency and amplitude for the MEPs and SEPs were significantly prolonged and decreased, respectively. 
These differences in the MEP and SEP parameters were larger than those for electroneuronography and BAEP. The different monitoring modalities used may themselves be associated with these differences. Moreover, in patients showing significant differences in the MEP and SEP monitoring, the TOF(\%) was observed to be lower by $75 \%$ than that in the other groups, even though the anesthesia regimen was similar. The latency of MEP increased more in muscles of the lower extremities. The differences in the degree of changes between muscles in the upper and lower extremities might be associated with the length of the corticospinal tract, where the longer pathway might be more sensitive to the factors in IONM. Unlike in MEPs, there is a short distance between the stimulation site and recording electrodes in electroneuronography and BAEP, which may be related to the minimum effect observed in these parameters. However, the SEP latency was higher in the median nerve than in the tibial nerve, which might indicate that the dorsal column of the sensory afferent tract was relatively less affected by the length of the tract. Neurophysiologists and neurosurgeon may use these differences between PONM and IONM as reference data, even if the PONM may not be performed in the facilities. In addition, using MEP/SEP, the anesthesiologic condition with low TOF or monitoring at the lower extremities are more vulnerable to changes in operative settings.

Additionally, in this study, patients with abnormal findings in PONM showed a higher degree of change from the IONM findings than those with normal PONM parameters, except for the SEPs in the tibial nerve (Table III). The latency and amplitude of the SEP of the median nerve was more prolonged and more decreased, respectively, in the group with abnormal PONM parameters compared to the group with normal ones. Additionally, a previous study reported that neurologic problems affected the success rates of MEPs in IONM (1). However, among the abnormal parameters obtained in PONM, the latency of SEPs in the tibial nerve showed a smaller increase in the IONM compared to the other parameters. We performed a further analysis by dividing the data based on the surgery site, i.e., the spine versus brain operation group. Interestingly, the latency of the SEP of the tibial nerve in brain surgery patients with abnormal PONM findings decreased during IONM, but increased during IONM in the spine surgery patients. The type of surgery did not affect the degree of change in other parameters. Additionally, Thirumala et al. (11) reported that demyelinated facial nerves might be less affected by intraoperative factors compared to healthy nerves, which might lead to false-negative findings. Therefore, it would be arbitrary to conclude that, compared with normal PONM parameters, abnormal PONM parameters show a greater change during IONM. We assume that these discrepancies may be due to differences in the measured nerve length and monitoring methods. Based on the results of this and previous studies, abnormal values in PONM seemed not to be predictable during IONM. These differences clinically implicate the importance of PONM for the early recognition of hidden neurologic deficits and predictable abnormality in IONM. In addition, the optimal indication of IONM for improving the success rate may be related to them, but specific patients' group and clinical settings should be required in future studies.
Unlike the abnormality of PONM values, which hemisphere was affected or unaffected in brain surgery patients did not induce any significant differences in IONM values (Table IV). The differences in the MEP latency and SEP amplitude between the affected and unaffected hemisphere were less than $1 \mathrm{msec}$ and $0.3 \mathrm{mV}$, respectively. Therefore, during brain operation, the electrophysiologic parameters might be less influenced by the hemisphere affected or unaffected, and the comparison of the parameters from the right and left hemispheres might be a reliable method of understanding the baseline values and changes in IONM.

As previously mentioned, the PONM and IONM parameters for electroneuronography and BAEP were not significantly different. The lack of significant change in the MEP latency and SEP amplitude might be attributed to the relatively small numbers of patients who underwent electroneuronography analyses. Additionally, the PONM values for latency in waves $\mathrm{I}$ and $\mathrm{V}$ in BAEP showed an insignificant difference, of less than $0.5 \mathrm{msec}$, from the IONM values. In previous studies, the failure rate of LSR in IONM was $5.5 \sim 12.7 \%$, although there was a response during PONM by all patients $(10,11)$. In our study, 34 patients showed compound motor action potentials in IONM for frontalis (34/38, 89.5\%) and 29 patients showed compound motor action potentials in IONM for oculi muscles $(29 / 38,76.3 \%)$ while 14 showed no response in IONM for oris $(14 / 38,36.8 \%)$ and 22 showed no response in IONM for mentalis $(22 / 38,57.9 \%)$, even though PONM values were obtained in all 38 patients. The fact that the amplitudes obtained during PONM were already very low could be the reason for the low success rate observed.

This study had several limitations. First, the sample size was small, especially for facial nerve monitoring, which could have affected the IONM results for electroneuronography and BAEP. However, MEPs and SEPs obtained during PONM and IONM showed statistically significant differences. Second, technical issues could have affected the data; for example, the different technicians and machines used for IONM and PONM. MEP stimulation was performed through transcranial magnetic stimulation using a Figure 8 coil before the operation and through electrical stimulation using a subdermal needle electrode during operation. Third, the anesthesia regimen differed depending on the operations and patients. Therefore, clinicians should consider establishing laboratory settings that reduce these differences between PONM and IONM and cooperate with anesthesiologists to use similar anesthesia regimens for further studies.

\section{CONCLUSION}

There are differences between PONM and IONM electrophysiologic values. Prolonged latency and decreased amplitude in MEPs, as well as prolonged latency in SEPs in the upper and lower extremities, were observed during IONM. Moreover, IONM of patients with abnormal PONM values showed significantly prolonged latency and lower amplitude. The affected or unaffected hemisphere in brain surgery patients did not make significant differences in the IONM parameters. Moreover, values obtained in relatively short monitoring 
sessions, such as facial nerve electroneuronography and auditory nerve BAEP, did not show any significant differences between PONM and IONM. These results can help clinicians better understand electrophysiologic parameters during operations.

\section{ACKNOWLEDGEMENTS}

This work was supported by the "Research Base Construction Fund Support Program" funded by Jeonbuk National University in 2018 and supported by Rehabailitation Institute of Neuromuscular Disease, Yonsei University College of Medicine research grant.

\section{- REFERENCES}

1. Chen X, Sterio D, Ming X, Para DD, Butusova M, Tong T, Beric A: Success rate of motor evoked potentials for intraoperative neurophysiologic monitoring: Effects of age, lesion location, and preoperative neurologic deficits. Journal of Clinical Neurophysiology 24(3):281-285, 2007

2. Chung J, Park W, Hong SH, Park JC, Ahn JS, Kwun BD, Lee SA, Kim S-H, Jeon JY: Intraoperative use of transcranial motor/sensory evoked potential monitoring in the clipping of intracranial aneurysms: Evaluation of false-positive and falsenegative cases. J Neurosurg 130(3):936-948, 2018

3. Daniel JW, Botelho RV, Buzetti JM, Dantas FR, Onishi F, Neto ER, Bertolini ED, Borgheresi MD, Joaquim AF: Intraoperative neurophysiological monitoring in spine surgery-a systematic review and meta-analysis response. Spine 43(13):E796-E797, 2018

4. Kim CH, Kong DS, Lee JA, Park K: The potential value of the disappearance of the lateral spread response during microvascular decompression for predicting the clinical outcome of hemifacial spasms: A prospective study. Neurosurgery 67(6):1581-1588, 2010

5. Kim WH, Lee J, Lee S, Park M, Park S, Seo D, Chung I: Comparison of motor-evoked potentials monitoring in response to transcranial electrical stimulation in subjects undergoing neurosurgery with partial vs no neuromuscular block. British Journal of Anaesthesia 110(4):567-576, 2013
6. Lall RR, Lall RR, Hauptman JS, Munoz C, Cybulski GR, Koski T, Ganju A, Fessler RG, Smith ZA: Intraoperative neurophysiological monitoring in spine surgery: Indications, efficacy, and role of the preoperative checklist. Neurosurgical Focus 33(5):E10, 2012

7. Lee SH, Park BJ, Shin HS, Park CK, Rhee BA, Lim YJ: Prognostic ability of intraoperative electromyographic monitoring during microvascular decompression for hemifacial spasm to predict lateral spread response outcome. Journal of Neurosurgery 126(2):391-396, 2017

8. Liveson JA, Ma DM: Laboratory reference for clinical neurophysiology. USA: Oxford University Press, 1992

9. Malcharek M, Loeffler S, Schiefer D, Manceur M, Sablotzki A, Gille J, Pilge S, Schneider G: Transcranial motor evoked potentials during anesthesia with desflurane versus propofol-A prospective randomized trial. Clinical Neurophysiology 126(9):1825-1832, 2015

10. Sekula RF Jr, Bhatia S, Frederickson AM, Jannetta PJ, Quigley MR, Small GA, Breisinger R: Utility of intraoperative electromyography in microvascular decompression for hemifacial spasm: A meta-analysis. Neurosurg Focus 27(4):E10, 2009

11. Thirumala PD, Shah AC, Nikonow TN, Habeych ME, Balzer JR, Crammond DJ, Burkhart L, Chang YF, Gardner P, Kassam AB, Horowitz MB: Microvascular decompression for hemifacial spasm: Evaluating outcome prognosticators including the value of intraoperative lateral spread response monitoring and clinical characteristics in 293 patients. J Clin Neurophysiol 28(1):56-66, 2011

12. Wang AC, Than KD, Etame AB, La Marca F, Park P: Impact of anesthesia on transcranial electric motor evoked potential monitoring during spine surgery: A review of the literature. Neurosurgical Focus 27(4):E7, 2009

13. Ziewacz JE, Berven SH, Mummaneni VP, Tu TH, Akinbo OC, Lyon R, Mummaneni PV: The design, development, and implementation of a checklist for intraoperative neuromonitoring changes. Neurosurgical Focus 33(5):E11, 2012 